\title{
Analyzing Rail Traffic Diversion Based on Machine Learning Technique considering Transportation Security
}

\author{
Jing Luo $\mathbb{i}$, ${ }^{1}$ Dai Zhou $\mathbb{D}^{1},{ }^{1}$ Wenjun $M a \mathbb{D}^{2,3}$ and Guohua Zhao ${ }^{4,5}$ \\ ${ }^{1}$ School of Naval Architecture, Ocean \& Civil Engineering, Shanghai Jiao Tong University, Shanghai 200240, China \\ ${ }^{2}$ School of Design, Shanghai Jiao Tong University, Shanghai 200240, China \\ ${ }^{3}$ China Institute of Urban Governance, Shanghai 200030, China \\ ${ }^{4}$ Shanghai Jiao Tong University Design \& Research Institute, Shanghai 200000, China \\ ${ }^{5}$ Consulting Department of PPP Research Center, Shanghai Jiao Tong University, Shanghai 200000, China \\ Correspondence should be addressed to Dai Zhou; zhoudai@sjtu.edu.cn
}

Received 9 September 2021; Accepted 20 January 2022; Published 22 February 2022

Academic Editor: Xinqiang Chen

Copyright $\odot 2022$ Jing Luo et al. This is an open access article distributed under the Creative Commons Attribution License, which permits unrestricted use, distribution, and reproduction in any medium, provided the original work is properly cited.

\begin{abstract}
Based on a stated preference survey, we comprehensively analyze the travel psychology of residents and the advantages and disadvantages of rail transit and conventional buses, travel time, travel cost, travel security, and vehicle comfort and investigate the relationship between the relevant influencing factors and the transition probability from rail transit to buses. A stochastic utility theory is introduced to describe the transfer behavior pertaining to travel modes, and a binary Logit model for diversion transfer is constructed. The decision tree is also used to predict the diversion transfer. Then, based on the large amount of travel willingness data obtained through the stated preference survey, a maximum likelihood estimation method is used to calibrate the parameters of the binary Logit model. The performance of the binary Logit proves to be better than that of the decision tree. Results show that the travel time most notably affects the passenger flow transfer, followed by the vehicle comfort. Finally, Guangzhou Rail Transit Line 3 is considered an example, and the diversion route planning and design are performed according to the constructed diversion transfer probability model to verify the effectiveness and practicability of the model. The research provides an effective theoretical basis and technical reference for other cities to perform rail traffic diversion planning. Based on these results, the following suggestions can be made: (1) the organization of public transportation routes, delivery volume, and travel speed outside should be improved; (2) undertaking combined operation of bus and rail transportation and integrated development is preferred; (3) the transportation management should focus on the comprehensive function development and hardware support of public transportation stations. The convenience and comfort of rail transit are closely related to the facilities and functions of the stations and their connections, which should be highly valued.
\end{abstract}

\section{Introduction}

In recent years, urban rail transit has developed rapidly in China. According to the difference in service scope, rail transit is generally divided into three categories, namely, national railway systems, intercity rail transit, and urban rail transit. As of the end of 2020, 45 cities have opened urban rail transit, with a total operating mileage of 7,978.19 kilometers [1]. At present, the scale of China's operating lines, lines under construction, and passenger flow ranks the first in the world. China has become a veritable "urban rail country." Urban rail transit is a large-volume rapid public transportation system with a wheel-rail operation system, and its main power source comes from electric energy. It is mainly responsible for barrier-free and short-distance travel, with light electric multiple units (EMUs) or trams as the transportation carrier, effectively alleviating the traffic pressure of dense passenger flow in the city. Urban rail transit generally has the advantages of large volume, fast speed, dense frequency, security and comfort, high punctuality, all-day operation, low freight, energy saving, and environmental protection [2]. From an operational perspective, in the future, we should fully use advanced technologies such as the Internet, 5G, big data, and artificial 
intelligence to promote operational informatization and intelligence. In addition, the application of intelligent customer service in subway stations should be actively explored to improve the convenience and satisfaction of passengers and reduce operating costs.

However, the current development stages of rail transit in various cities are relatively different; thus, the problems they face are also different. For example, in the urban rail transit development initial stage (generally referring to an average daily passenger flow of less than 1 million), rail transit often has only one line or a few lines in operation, the line scale is small, and the attraction to people is insufficiently high. It does not show the benefits of networked operations, resulting in a relatively low percentage of commuting passenger flow during weekdays and a relatively high percentage of leisure and entertainment passenger flow on weekends [3]. The passenger flow on weekends is often larger than that during working days $[4,5]$. When the development of urban rail transit reaches an intermediate level (generally, the average daily passenger flow is between 1 million and 5 million), the commuting passenger flow begins to increase significantly and the passenger flow on weekends follows a downward trend [6]. The network accessibility of cities that have entered a mature operation stage (generally referring to an average daily passenger flow of more than 10 million) gradually improves. Furthermore, commuting passenger flow accounts for a high proportion and weekend passenger flow has dropped significantly [7]. Among them, Friday may create a "peak day of passenger flow" during the week due to the overlap of commuting passenger flow after work and the subsequent leisure and entertainment passenger flow. Therefore, the problem of congestion in the carriages becomes increasingly prominent. For the entire year of 2019, the average daily urban rail transit passenger flow of 28 major cities across the country was 72.77 million. The top five cities were Beijing, Shanghai, Guangzhou, Hong Kong, and Shenzhen, and the average daily urban rail passenger flow was $10.82,10.6,9.03,8.68$, and 5.49 million. The findings evidently show the differences in rail transit passenger flow among different cities. The top four cities account for approximately 53\% of China's passenger flow, and the top five cities account for approximately $61 \%$ of China's passenger flow [8]. Taking Guangzhou as an example, the congested section of rail transit shows a trend of spreading out with the Zhujiang New Town (urban CBD area) as the center. During the peak period, the full load rate of most sections of Lines 3 and 5 exceeds 100\%, and the flow of some stations is controlled. The passenger flow control has reduced the service level of the rail transit. Rail transitsupported urban growth can be an effective strategy for countering automobile dependence typically associated with the population increase, economic development, and physical expansion of large cities [9]. However, some studies have found that the development of rail transit often has a negative impact on social equity because public transport institutions tend to allocate more available subsidies to the construction and operation of the railway system while abandoning the successful policies of improving public transport services and reducing public transport fares. The poorest urban residents mainly benefit from buses rather than rail transit, and the effect of using rail transit to achieve environmental goals is often unclear $[10,11]$. Moreover, Wang et al. found that the opening of Chengdu Metro Line 4 is unlikely to improve air quality [12].

Compared with rail transit, buses and trams have flexible advantages [13]. They can go deep into areas not covered by rail transit, provide citizens with "door-to-door" travel services, and create a more comfortable riding environment than rail transit [14]. Electrification, new energy, and cleaning of public transport have become irreversible trends, which can effectively promote the solution of energy security and urban air pollution [15]. These approaches can reduce climate change related to fossil fuels to a certain extent [16]. Therefore, in the megacities, to effectively alleviate the passenger flow pressure, travel modes should be diverted from rail transit to buses, and the coordinated development of public transportation should be realized [17, 18]. Beijing, Shanghai, Guangzhou, Shenzhen, Suzhou, and other cities have successively implemented transfer preferential policies to promote rail transit and bus transfers. In the initial stage of rail transit (generally, urban rail transit accounts for less than $30 \%$ of public transportation), the network operation has not yet been fully constructed, rail transit has just been introduced as a new mode of transportation, and the public transportation is still borne by buses. In the development period of urban rail transit (generally, urban rail transit accounts for between $30 \%$ and $50 \%$ of public transportation), the auxiliary role of rail transit in urban public transportation has initially appeared, and buses still play the main role in public transportation. In the mature period of urban rail transit (generally, urban rail transit accounts for more than $50 \%$ of public transportation), the dominant position of rail transit slowly emerges.

In the development of public transportation, in addition to the coordinated development of bus and urban rail transit, a certain mutual substitution problem exists between the two; that is, travelers may transfer from buses to rail transit or from rail transit to buses. Travelers' preference of buses or rail transit can be attributed to the travel modes [19-21]. The variables that affect the residents' preference of travel modes can be divided into two major categories, namely, manifest variables and latent variables. Manifest variables include personal attributes, family attributes, travel attributes, and regional attributes [22]. Personal attributes mainly indicate gender, age, occupation, education level, income, and whether to have a bus card or hold a driver's license. Family attributes mainly refer to the number of bicycles, the number of electric vehicles, the number of cars, and whether schoolage children exist. Travel attributes mainly indicate the purpose of the trip, the distance of the trip, the cost of the trip, and the time of the trip. Regional attributes refer to the economic level of the region, the density of residents, and the degree of perfection of transportation facilities [23]. The latent variables mainly include the risk attitude (risk aversion, risk neutrality, and risk preference), travel habits, and travel experience of travelers [24].

Another important factor that influences travel mode preference is travel security. Reports of travel security 
incidents that have occurred in recent years have sounded the alarm for people and indirectly affected their transfer behavior. On July 20, 2021, Zhengzhou City, Henan Province, continued to experience extreme heavy rains, causing serious water accumulation in the Wulongkou parking lot of Metro Line 5 and its surrounding areas. A total of 14 passengers were killed in the accident. The incident caused the entire society to consider the use of public transportation in urban emergencies; it also affected people's preference for rail transportation in certain situations.

During the COVID-19 pandemic, travel security has become a more important factor in residents' travel modes preference [25]. Subways are underground with limited fresh air; thus, various works on analyzing potential risks to health have been conducted, thereby intensively increasing respiratory-related diseases [26]. The perception of safety in public transport, characterized by the social distancing and sanitization measures, determines the willingness of people toward selecting public transit systems [27]. Khadem et al. [28] studied the impact of this pandemic on the transportation of Tehran, the capital of Iran, by two Logit models to determine how people shift from the subway to other modes and from other modes to private cars. They found that the number of work trips had a negative impact, and income had a positive impact on shifting away from the subway. Moreover, the results from Toronto and Germany indicate that the importance of public transit has declined during the pandemic, whereas the roles of private vehicles and active modes have become more prominent $[29,30]$. $\mathrm{Hu}$ et al. [31] used the SP survey method to conduct travel surveys in Zhongshan City, Guangdong Province, and divided the survey period into three stages, namely, the early, middle, and the latter stages of the epidemic, that is, from December 2019 to January 20, 2020, January 21, 2020, to February 23, 2020, and after February 24, 2020, respectively. The Nested Logit (NL) model was used to analyze the key factors that affect residents' travel during COVID-19. The research results show that, in the early stage of COVID-19, whether travelers own a car, economic status, travel purpose, travel time, and travel distance have a significant impact on selecting the public transportation mode. In the middle stage of COVID-19, age and travel purpose have a significant impact on selecting public transportation mode. The reason may be that, with the outbreak of COVID-19 on a large scale, due to security considerations, control measures such as suspension of work, suspension of classes, and home isolation have been implemented, and the travel of residents has changed accordingly [10]. In the later stage of COVID-19, the age of travelers, whether they own a car, economic status, and travel purpose have the most significant impact on public transportation mode preference, whereas travel time and travel distance no longer have a significant impact. Jiang et al. [32] collected information on travel frequency and transportation modes of 8,330 residents in 31 provinces (autonomous regions and municipalities) in China from February 25 to March 14, 2020, and analyzed its influencing factors. The research results show that, during COVID-19, the travel frequency and mode of transportation of Chinese residents may be affected by demographic variables, such as gender, age, urban and rural areas, districts, and the situation of suspected/confirmed cases in neighborhoods. The travel frequency and transportation modes of travelers in Tibet Autonomous Region, Qinghai Province, and other regions have relatively little changes due to COVID-19. The travel frequency and transportation modes of residents in Hubei Province have the largest changes compared with the usual. In addition, the proportion of Chinese adult residents, who prefer to travel by foot and car (taxi and private car), is much higher than usual, whereas the proportion of public transportation has decreased compared with the usual. This finding is mainly because residents are concerned about COVID-19 when they choose public transportation and the traffic management department's control of public transportation. For example, during COVID-19, Shanghai has successively introduced strict sanitation management measures in rail transit, buses, taxis, and other transportation modes, especially the prevention and control of rail transit epidemic. During the first-level response period, Shanghai rail transit implemented flow limiting measures at key stations during the morning rush hour; some subway lines were operated until 21:00 in advance and gradually recovered until the end of April 2020 [33]. In 2020 and a long time in the future, human society has entered a period of coexistence with COVID-19.

The existing research on passenger flow transfer primarily focused on the feasibility of predicting the passenger flow transfer from buses to rail transit. The main prediction models include the binary Logit $(\mathrm{BL})$ model [8], multinomial Logit (MNL) model [31], NL model [32], and mixed Logit (ML) model [33]. For example, Lv [34] used the SP survey method to collect data, constructed a Logit model of passenger flow transfer from buses to rail transit, conducted a sensitivity analysis of travel time and travel cost, and predicted the impact of rail transit fare level on subway passenger flow transfer in Xi'an with quantitative analysis of the competitiveness of existing buses and rail transit. He proposed ways to influence traffic policies based on analytical flexibility analysis. The research results show that travel costs, travel distance, and travel time are the most important factors affecting the transfer of rail transit passenger flow in Xi'an. Zhang [35] considered Xi' an Metro Line 2 as an example, used the SP survey method to obtain basic data, constructed an MNL model, and made a detailed analysis of various influencing factors and their impact levels in the transfer of buses to rail transit. The transfer rate of buses to rail transit in the corridor of Xi'an Metro Line 2 is obtained. Cao et al. [36] collected the SP survey data of the travel mode selection behavior of residents in Urumqi, considered factors such as traffic information and travel mode selection habits of residents, and constructed an NL model to predict the passenger flow transfer problem after the completion of rail transit. The results of the study show that, after the completion of rail transit Lines 1 and 2, they can bear $34.7 \%$ of residents' trips. Residents, who usually prefer taxis, tend to move to rail transit, and the proportion of buses and taxis is greatly reduced. The reduction in the proportion of private car trips is the smallest. 
In summary, considerable research on the transfer of passenger flow from buses to newly built rail transit systems has been conducted. This study represents a transfer from low-quality and low-speed modes to high-quality and highspeed modes. The problem involving the diversion of the passenger flow from rail transit to buses is considerably different from the transfer problem. Such a transfer may contribute to a more coordinated, high-efficiency, and sustainable multimodal transportation system. In addition, it is necessary to explore the effect of transportation security on the transfer during the pandemic of COVID-19. Although many cities have attempted to realize diversion, the implementation has not been effective due to the lack of theoretical support. Therefore, we comprehensively consider the relative factors that affect the travel mode choice of passengers and the relative advantages of buses. Based on stochastic utility theory, a rail transit diversion probability model is constructed to provide a theoretical basis and technical support for the planning of rail transit diversion routes in related cities.

\section{Data Collection and Analysis}

Rail transit involves a higher speed and punctuality than buses; thus, it has gradually become the primary choice for most passengers. Therefore, diverting the passenger flow of rail transit and changing the habits of passengers are challenging. To grasp the travel psychology and behavior of rail transit passengers, a travel willingness survey should be conducted.

\subsection{Travel Willingness Survey. The stated preference survey} is a highly effective travel willingness survey method that is currently widely used in domestic and international settings $[34,35,37]$. This method is used to investigate whether rail transit passengers are willing to transfer to buses.

To address the prominent problem of the high passenger flow demand pertaining to the rail transit lines connecting the city center and suburbs, we focus on the problem of diversion in long-distance commuting. Guangzhou Rail Transit Line 3, Line 3 Northern Extension Line, and Line 5 are selected to conduct an on-site survey of the travel willingness. The planning and operation of the line must satisfy certain passenger flow conditions. Therefore, whether a certain amount of passenger flow exists between two rail transit stations should be identified to evaluate whether the diversion line can be planned in the surrounding communities of the two rail transit stations. According to the analysis of the OD pairs of the rail transit stations, there exist 14 stations with relatively large passenger flow connections in the peripheral sections of the 3 rail transit lines. Therefore, these 14 stations are selected for the questionnaire survey. These survey sites are Shiqiao, Hanxi Changlong, Dashi, Xiajiao, and Datang for Line 3; Longgui, Jiahe Wanggang, Yongtai, Tonghe, and Jingxi Nanfang Hospital for Line 3 Northern Extension Line; Dashadi, Sanxi, Chebei South, and Keyun Road for Line 5.
According to the research results of the residents' travel psychology, the main factors that affect the residents' travel choices include timeliness [36], comfort [38], security [39], economy [40], and convenience [41]. Moreover, the pain points associated with rail traffic congestion and transfers and the advantages of buses are considered. Finally, the contents of the survey are designed, including the personal basic attributes, travel time, travel costs, travel security, vehicle comfort, transfer characteristics, and the transfer choice under different scenarios. A uniform design method is adopted to design the SP survey questionnaire to ensure the accuracy of the data collection and minimize the number of questions answered by the respondents. In addition, combinations of different times, costs, vehicle types, and transfer types are considered for the survey.

Travel time includes two factors, namely, bus travel time and rail transit travel time. Travel costs also include bus travel costs and rail transit travel costs. Travel security also includes bus travel security and rail transit travel security. Six factors, including travel time, travel cost, and travel security, are designed to be eleven levels, considering the complexity of the survey and the reliability of the data obtained. The vehicle comfort (with 0 and 1 indicating ordinary and commercial buses, resp.) and the transfer feature (with 0 and 1 indicating no transfer and transfer, resp.) are two dummy variables designed to be two levels. Therefore, a mixed homogeneous design survey plan design table with six factors at eleven levels and two factors at two levels should be selected.

Furthermore, to minimize the number of questions in the questionnaire, the traditional uniform design scheme is modified. The travel time of buses and rail transit is expressed as the ratio of the travel time of buses to that of rail transit. Similarly, the travel costs of buses and rail transit are uniformly expressed as the ratio of bus travel costs to rail transit costs. The travel security of buses and rail transit is uniformly expressed in terms of the travel security ratio of buses to rail transit. Therefore, the original eight-factor plan is adjusted to a five-factor plan, and a mixed uniform design survey plan with three factors at eleven levels and two factors at two levels should be selected.

According to the uniform design standardization table, the most similar table to the mixed uniform design table of three factors eleven levels and two factors two levels is $U_{11}$ $\left(11^{6}\right)$, whose uniform design table and usage table are shown in Tables 1 and 2, respectively. According to the usage table, when only five factors are available, columns 1 , $2,3,4$, and 5 should be selected. Then, 1-11 in the fourth and fifth columns should be transferred as 0 and 1 of the vehicle comfort and transfer characteristics. Specifically, $1-5$ correspond to $0,6-10$ correspond to 1 , and 11 corresponds to 0 and 1 with a $50 \%$ probability. Thus, the uniform design plan of the experiment on the influence factors of the selection of buses and urban rail transit is shown in Table 3.

The survey was carried out from May 26 to May 29, 2020, and a total of 2,275 valid samples were obtained. Through 
TABLE 1: $U_{11}\left(11^{6}\right)$ uniform design table.

\begin{tabular}{|c|c|c|c|c|c|c|}
\hline & 1 & 2 & 3 & 4 & 5 & 6 \\
\hline 1 & 1 & 2 & 3 & 5 & 7 & 10 \\
\hline 2 & 2 & 4 & 6 & 10 & 3 & 9 \\
\hline 3 & 3 & 6 & 9 & 4 & 10 & 8 \\
\hline 4 & 4 & 8 & 1 & 9 & 6 & 7 \\
\hline 5 & 5 & 10 & 4 & 3 & 2 & 6 \\
\hline 6 & 6 & 1 & 7 & 8 & 9 & 5 \\
\hline 7 & 7 & 3 & 10 & 2 & 5 & 4 \\
\hline 8 & 8 & 5 & 2 & 7 & 1 & 3 \\
\hline 9 & 9 & 7 & 5 & 1 & 8 & 2 \\
\hline 10 & 10 & 9 & 8 & 6 & 4 & 1 \\
\hline 11 & 11 & 11 & 11 & 11 & 11 & 11 \\
\hline
\end{tabular}

TABLE 2: $U_{11}\left(11^{6}\right)$ use table.

\begin{tabular}{|c|c|c|c|c|c|c|}
\hline Number of factors & \multicolumn{6}{|c|}{ Column number } \\
\hline 2 & 1 & 5 & & & & \\
\hline 3 & 1 & 4 & 5 & & & \\
\hline 4 & 1 & 3 & 4 & 5 & & \\
\hline 5 & 1 & 2 & 3 & 4 & 5 & \\
\hline 6 & 1 & 2 & 3 & 4 & 5 & 6 \\
\hline
\end{tabular}

TABle 3: Stated preference survey uniform design scheme for bus versus rail transit.

\begin{tabular}{|c|c|c|c|c|c|}
\hline & 1 & 2 & 3 & 4 & 5 \\
\hline 1 & $0.5(1)$ & $0.6(2)$ & $0.7(3)$ & $0(5)$ & $1(7)$ \\
\hline 2 & $0.6(2)$ & $0.8(4)$ & $1.0(6)$ & $1(10)$ & $0(3)$ \\
\hline 3 & $0.7(3)$ & $1.0(6)$ & $1.3(9)$ & $0(4)$ & $1(10)$ \\
\hline 4 & $0.8(4)$ & $1.2(8)$ & $0.5(1)$ & $1(9)$ & $1(6)$ \\
\hline 5 & $0.9(5)$ & $1.4(10)$ & $0.8(4)$ & $0(3)$ & $0(2)$ \\
\hline 6 & $1.0(6)$ & $0.5(1)$ & $1.1(7)$ & $1(8)$ & $1(9)$ \\
\hline 7 & $1.1(7)$ & $0.7(3)$ & $1.4(10)$ & $0(2)$ & $0(5)$ \\
\hline 8 & $1.2(8)$ & $0.9(5)$ & $0.6(2)$ & $1(7)$ & $0(1)$ \\
\hline 9 & $1.3(9)$ & $1.1(7)$ & $0.9(5)$ & $0(1)$ & $1(8)$ \\
\hline 10 & $1.4(10)$ & $1.3(9)$ & $1.2(8)$ & $1(6)$ & $0(4)$ \\
\hline 11 & $1.5(11)$ & $1.5(11)$ & $1.5(11)$ & $0(11)$ & $1(11)$ \\
\hline
\end{tabular}

Note: in the first row, 1, 2, 3, 4, and 5 represent travel time ratio, travel cost ratio, travel security ratio, vehicle comfort, and transfer characteristics, respectively.

TABLE 4: Correlation between transfer probability and related factors.

\begin{tabular}{|c|c|c|c|c|c|c|}
\hline & Relevant factor & $\begin{array}{c}\text { Travel time } \\
\text { ratio }\end{array}$ & $\begin{array}{c}\text { Travel cost } \\
\text { ratio } \\
\end{array}$ & $\begin{array}{c}\text { Travel security } \\
\text { ratio }\end{array}$ & $\begin{array}{l}\text { Vehicle } \\
\text { comfort }\end{array}$ & $\begin{array}{c}\text { Transfer } \\
\text { characteristics }\end{array}$ \\
\hline \multirow{4}{*}{$\begin{array}{l}\text { Transition } \\
\text { probability }\end{array}$} & Pearson correlation & -0.726 & -0.467 & 0.617 & 0.618 & 0.354 \\
\hline & Significance (bilateral) & 0.000 & 0.000 & 0.000 & 0.000 & 0.000 \\
\hline & $\begin{array}{c}\text { Sum of squares and cross } \\
\text { products }\end{array}$ & -14.281 & -11.086 & 13.238 & 12.779 & 13.043 \\
\hline & Covariance & -0.062 & -0.048 & 0.054 & 0.094 & 0.073 \\
\hline
\end{tabular}

statistical analysis of the survey data, the correlation between the transition probability of rail transit to bus and the relevant influencing factors is shown in Table 4.

2.2. Data Characteristic Analysis. According to the survey data, rail transit passenger transfer is affected by many factors and thus represents a multifactor selection process. The transfer probability and travel time, travel cost, travel security, vehicle comfort, and transfer characteristics present a relatively scattered scatter-diagram relationship. Through the classification and average calculation of the data, the following relevant characteristics are obtained.

2.2.1. Relationship between the Transition Probability and Travel Time. According to the correlation, the transition probability is significantly affected by the travel time ratio of buses to rail transit (referred to as the travel time ratio). The correlation coefficient is -0.726 , indicating that this factor is the most relevant among the five influencing factors. This finding shows that passengers prioritize travel time when 
choosing a travel mode. According to the relationship diagram (Figure 1), the transition probability and travel time ratio follow logarithmic distributions. The transition probability decreases as the travel time ratio increases. When the travel time ratio increases to 1.3 , the transition probability is almost zero, indicating that the travel time of buses is increased to a certain extent, and passengers do not choose to divert.

2.2.2. Relationship between the Transition Probability and Travel Costs. According to the correlation, the travel cost ratio of buses to rail transit (referred to as the travel cost ratio in the following sections) exerts a less significant influence on the transition probability than the travel time ratio. The correlation coefficient is -0.467 , which represents a medium degree of correlation. This finding shows that passengers are not as sensitive to travel costs compared with travel time. According to the relationship diagram (Figure 2), the overall transition probability decreases as the travel cost ratio increases, but the tendency has certain volatility. This finding also shows that passengers are less sensitive to travel costs than travel time.

\subsubsection{Relationship between Transition Probability and Travel} Security. From the perspective of relevance, the impact of the travel security ratio between buses and rail transit (hereinafter referred to as the travel cost ratio) on the transition probability is relatively strong, with a correlation coefficient of 0.617 , indicating that passengers focus more on travel security. The relationship diagram (Figure 3 ) shows that the transition probability increases as the travel security ratio increases and the trend is relatively evident. This finding also shows that passengers are more sensitive to travel security in terms of travel costs, but less sensitive than travel time.

2.2.4. Relationship between the Transition Probability and Vehicle Comfort. In the travel willingness survey, two types of buses are considered, specifically, ordinary buses that allow for standing and commercial buses with one seat per person. The correlation coefficient between the vehicle comfort and transition probability is 0.618 , which indicates a strong correlation. According to the relationship diagram (Figure 4), among the transferred passengers, commercial and ordinary buses account for $78.4 \%$ and $21.6 \%$, respectively. This finding shows that vehicle comfort is important in selecting travel mode.

2.2.5. Relationship between the Transition Probability and Transfer Characteristics. The occurrence of a transfer in the travel chain is directly related to the convenience of travel. Some passengers may perform bus interchange to connect to rail transit due to a wide range in the outer area. Therefore, in the survey, the passengers are divided into two categories, namely, those who need to make a transfer trip of "bus + rail" and those who make a nontransfer trip. The survey results show that the correlation coefficient between the transfer characteristics and transition probability is 0.354 , which indicates a weak correlation. Among the passengers in the flow, transfer and nontransfer passengers account for $73.7 \%$

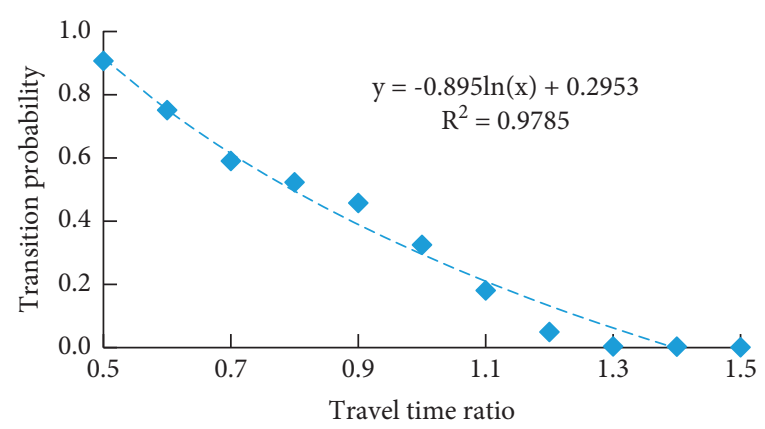

FIGURE 1: Relationship between transition probability and travel time ratio.

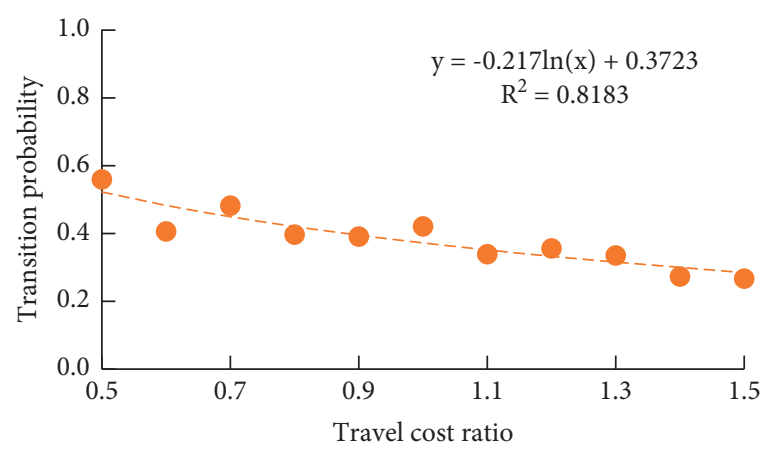

FIGURE 2: Relationship between transfer probability and travel cost ratio.

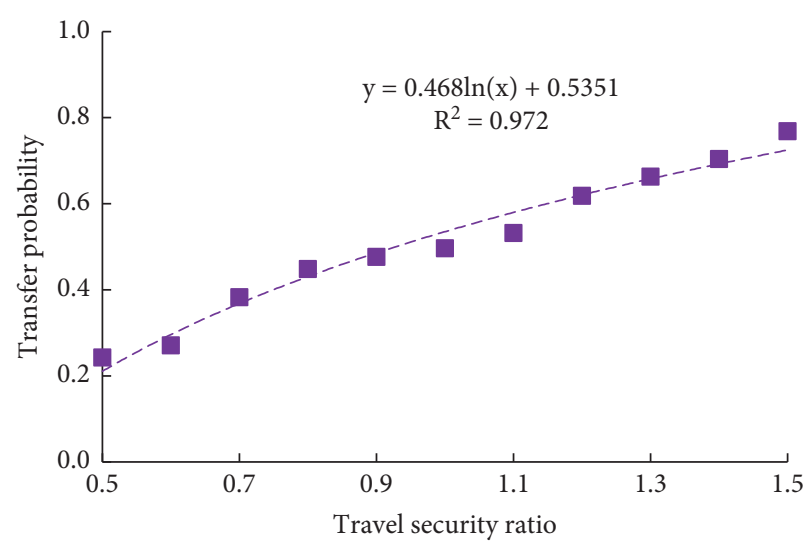

FIgURE 3: Relationship between transfer probability and travel security ratio.

and $26.3 \%$, respectively. This finding shows that although transfer characteristics are not a key factor affecting the transfer probability, transfer passengers account for a larger proportion. The relationship between the transition probability and transfer characteristics is shown in Figure 5.

\section{Constructions of the Diversion Probability Model}

3.1. Conceptual Model of the Diversion Probability. Whether rail transit passengers choose to prefer buses or continue to use rail transit is a typical binary choice problem. 


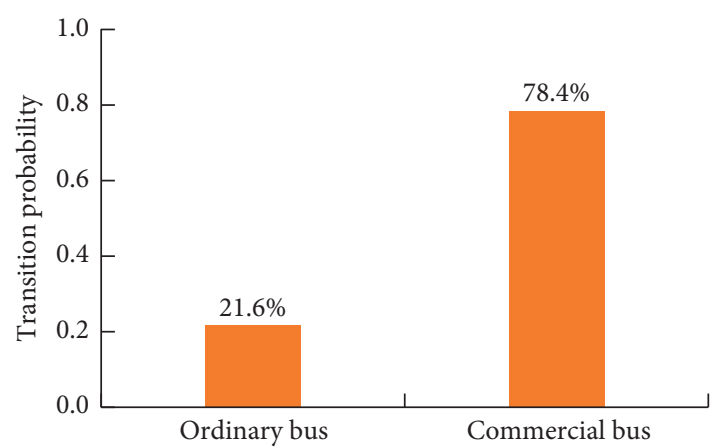

FIgURE 4: Relationship between transition probability and vehicle comfort.

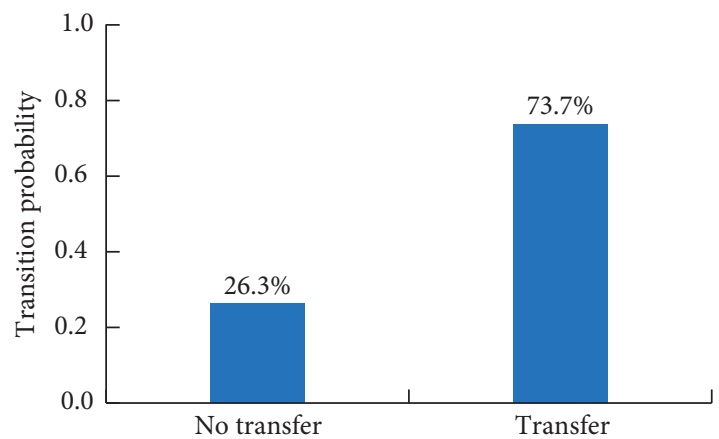

FIGURE 5: Relationship between transfer probability and transfer characteristics.

The decision-making process of the travel mode selection is affected by many factors, the essence of which is the comprehensive utility comparison between the two modes. The selection of rail transit passengers to divert to buses may be represented using the BL model.

According to the random utility theory, the travel utility of rail transit and buses is random and may be expressed using several determinants that affect the selection and a random error term. The utility function of the passenger's preference of transportation mode is expressed as follows:

$$
U_{\text {in }}=V_{\text {in }}+\varepsilon_{\text {in }}
$$

where $U_{\text {in }}$ is the total utility of passenger $n$ selecting transportation mode $i ; V_{\text {in }}$ is the systematic component of the utility function; $\varepsilon_{\text {in }}$ is a random term for utility, which follows the Gumbel distribution.

The systematic utility function is expressed as a linear function as follows:

$$
V_{\text {in }}=\alpha X_{\text {in }}+\beta v_{n},
$$

where $X_{\text {in }}$ is the variable that affects the transportation mode preference; $v_{n}$ is the variable specific to individual passengers; $\alpha$ and $\beta$ are variable parameters. follows:

The probability that passengers opt to divert to a bus is as

$$
P_{\text {bus }}=\frac{\exp V_{\text {bus }}}{\exp V_{\text {bus }}+\exp V_{\text {metro }}}
$$

where $P_{\text {bus }}$ is the probability that passengers divert to buses from rail transit.

3.2. Selection of Model Variables. Integrating the domestic and international research regarding the variables influencing the travel mode option and the results of the travel willingness survey, the following variables are selected as the input variables of the model, and the following definitions are provided.

Travel Time Difference between Buses and Rail Transit. A smaller value of the travel time of buses relative to rail transit corresponds to a larger number of passengers that choose buses; otherwise, most passengers continue to use rail transit. This indicator is an inverse indicator, and it is expressed as follows:

$$
\varphi_{t}=T_{\text {bus }}-T_{\text {metro }},
$$

where $\varphi_{t}$ is the travel time difference between bus and rail transit and $T_{\text {bus }}$ and $T_{\text {metro }}$ represent the travel time for buses (min) and rail transit (min), respectively.

Travel Cost Difference between Buses and Rail Transit. A lower travel cost of buses relative to rail transit corresponds to a larger number of passengers that select buses; otherwise, most passengers continue to use rail transit. This indicator is an inverse indicator, expressed as follows:

$$
\phi_{\text {cost }}=C_{\text {bus }}-C_{\text {metro }}
$$

where $\phi_{\text {cost }}$ is the travel cost difference between buses and rail transit and $C_{\text {bus }}$ and $C_{\text {metro }}$ represent the travel cost of buses (Yuan) and rail transit (Yuan), respectively.

Travel Security Difference between Buses and Rail Transit. The higher travel security of bus relative to rail transit indicates that more passengers choose bus; otherwise, most of them continue to take rail transit. This indicator is a positive indicator, expressed as follows:

$$
\delta_{\text {security }}=\delta_{\text {bus }}-\delta_{\text {metro }} \text {, }
$$

where $\delta_{\text {security }}$ is the travel security difference between buses and rail transit; $\delta_{\text {bus }}$ and $\delta_{\text {metro }}$ represent the travel security of buses and rail transit, respectively.

Vehicle Comfort. The comfort of buses is a key factor that affects travel choice. A higher vehicle comfort corresponds to a larger number of passengers that choose buses. Conversely, higher crowding of buses corresponds to a lower probability of passenger transfer. This indicator is positive, and it is expressed as a dummy variable as follows:

$$
\gamma_{\text {bus }}= \begin{cases}1, & \text { when a bus is crowded, } \\ 0, & \text { when a bus is uncrowded, }\end{cases}
$$

where $\gamma_{\text {bus }}$ is the dummy variable of bus comfort, with 0 and 1 indicating ordinary and commercial buses, respectively. 
Transfer Characteristics. Transfer is a key factor affecting travel convenience. If the rail transit is more convenient, then fewer passengers transfer to a bus; otherwise, the passengers would consider transferring to a bus. This indicator is positive and represented by a dummy variable as follows:

$$
\lambda_{\text {bus }}= \begin{cases}1, & \text { when a transfer is needed, } \\ 0, & \text { when a transfer is not required, }\end{cases}
$$

where $\lambda_{\text {bus }}$ is the dummy variable of transfer characteristics, with 0 and 1 indicating no transfer and transfer, respectively.

Based on the preliminary selection of the input variables of the transfer model, stepwise regression and factor analyses are performed to analyze the correlation of the input variables. The results show that the input variables of the five models are highly independent of one another. Therefore, these variables are adopted in the transfer model. The input variables are shown in Table 5.

3.3. Model Parameter Calibration. Among the existing parameter calibration methods, the two most commonly used methods are the least square method and the maximum likelihood estimation method. The least square method identifies the best function match of the data by minimizing the sum of squares of the error. When the model is a classic linear regression model, the least square estimator exhibits a high estimation accuracy. Thus, the least square method is mainly used for regression coefficients. The maximum likelihood estimation method uses the known sample results to reverse the highest likelihood (maximum probability) to obtain the parameter value of the current sample as the estimated value of the parameter. Both methods can be applied in many cases. The Logit model is a nonlinear function; thus, the least square method is more complicated in estimating the parameters. Therefore, the maximum likelihood estimation method is most commonly used by the academic community to calibrate Logit model parameters. The method has been widely embedded in statistical analysis software. Therefore, the maximum likelihood estimation method is used to calibrate the parameters of the constructed transfer model. Based on the large amount of SP data obtained from the survey, EViews software is used to estimate the parameters of the diversion model. The parameter estimation results of the transfer model are shown in Table 6.

According to the parameter calibration results, the constructed diversion probability model for rail transit to buses is as follows:

$$
P_{\text {in }}=\frac{1}{1+\exp \left(48.30+7.58 \varphi_{t}+2.97 \phi_{\text {cost }}-5.25 \delta_{\text {security }}-1.83 \gamma_{\text {bus }}-4.19 \lambda_{\text {bus }}\right)}
$$

3.4. Decision Tree. Decision tree is a classical classification algorithm of supervised learning in machine learning. Its tree structure is intuitive and easy to implement and has strong interpretability. It can be used to discover the data features in the database and extract patterns. This approach is very important for classification modeling.

The decision tree model can model data sets with many attributes. The nonlinear relationship between the relevant influencing factors and the transition from rail transit to buses is analyzed. Meanwhile, the decision tree belongs to the white box model, and the entire calculation process is visible, very intuitive, and easy to understand and explain. The data preprocessing stage is also relatively simple and can deal with missing data. The decision tree model can better judge the probability of residents' transition from rail transit to buses and identify the key factors affecting residents' transfer. According to results from the performance comparison between the binary Logit and decision tree in Table 7, the performance of the binary Logit is evidently better than that of the decision tree. This result may be due to the fact that the decision tree is sensitive to outliers, and its robustness should be further improved.

\section{Case Calculation Analysis}

Guangzhou Rail Transit Line 3 is a typical line that connects the central and suburban areas. This line is the most crowded line in Guangzhou, with a full load rate of $141 \%$ in peak hours. Shiqiao Station of Line 3 is considered an example. On the basis of the established diversion probability model, diversion route planning and design are performed.

\subsection{Big Data Mining}

4.1.1. Mobile Phone Data Mining. We collected one month's data on the network signaling data of China Unicom. The signaling data covers the user's real-time location information with the latitude and longitude of the base station tower. The accuracy of the urban data is approximately 250 meters. In addition, China Unicom has summarized and analyzed the basic information and behavioral data of users across the network, identified the attributes and behavioral preferences of users, and formed a 360-degree user portrait of users based on label information. More than 3,700 user tags of nine categories are available. The average daily number of users is 6.2 million, the daily average number of original signaling items is 2.1 billion, and the total number of monthly original signaling items is 64.3 billion. The data accuracy and scale satisfy the requirements of the program design. The distribution of China Unicom's base stations is shown in Figure 6.

After collecting each user's daily mobile phone signaling, a time-based user trajectory sequence is constructed, and the user's trajectory point is identified through mobile phone 
TABle 5: Feature index selection.

\begin{tabular}{lcc}
\hline Attributes & Characteristic index & Indicator direction \\
\hline Timeliness & Travel time difference & - \\
Economy & Travel cost difference & - \\
Security & Travel security difference & + \\
Comfort & Vehicle comfort & + \\
Convenience & Transfer characteristics & + \\
\hline
\end{tabular}

TABLE 6: Calibration results of the transfer Logit model parameters.

\begin{tabular}{lccc}
\hline Variable & Parameter & Standard error & $z$-statistic \\
\hline$C$ & -48.30 & 0.06234 & 4.26 \\
$\varphi_{t}$ & -7.58 & 0.02561 & -4.61 \\
$\varphi_{\text {cost }}$ & -2.97 & 0.03512 & -3.29 \\
$\delta_{\text {security }}$ & 5.25 & 0.02843 & 3.51 \\
$\gamma_{\text {bus }}$ & 1.83 & 0.04813 & 2.51 \\
$\lambda_{\text {bus }}$ & 4.19 & 0.04543 & 2.04 \\
\hline
\end{tabular}

TABle 7: Performance comparison between the binary Logit and decision tree.

\begin{tabular}{lccc}
\hline Methods & Precision & Recall & Accuracy \\
\hline Binary Logit & 0.8595 & 0.9322 & 0.9421 \\
Decision tree & 0.8054 & 0.9123 & 0.9190 \\
\hline
\end{tabular}

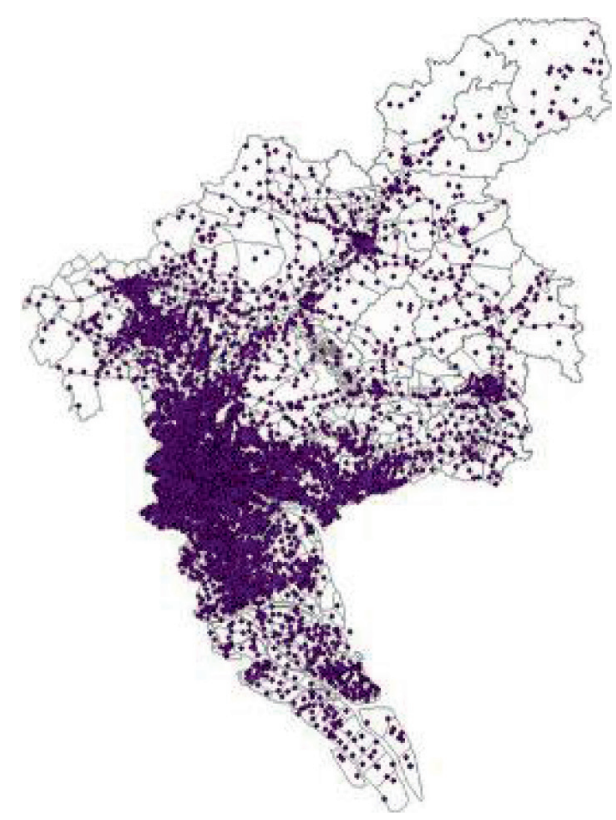

Figure 6: Distribution of China Unicom's base stations in Guangzhou.

signaling. After constructing a time-based user travel trajectory sequence, combined with the longitude and latitude coordinates of base stations, the user's trajectory points are combined, and the stationary point judgment algorithm can be used to obtain the user's effective stay point. Among them, the effective stay trajectory analysis is mainly based on the effective trajectory point, using the constraints of the distance position between the base stations to complete the calculation of the effective stay point. After analyzing and obtaining the mobile phone travel OD, we can dig out the trajectory of each OD to identify a series of information, such as the departure subway station, the passing subway station, and the destination subway station of each subway trip. This set of information is used to analyze the spatial distribution of rail transit passenger flow.

4.1.2. IC Card Data Mining. In the IC card analysis process, this work focuses on obtaining the OD stops for bus passengers. The bus OD estimation analysis is mainly based on the assumption that urban residents' bus travel chains are closed. In accordance with the interrelationship between bus stops, bus routes, and card swiping information and the last drop-off site information, the information about getting on and off the buses for passengers under the one-ticket system of the public transportation system is obtained. Through the transfer identification between buses and rail transit, the scale, space, and time distribution of the transfer passenger flow are grasped. The specific analysis process is shown in Figure 7.

4.2. Characteristics of Site Passenger Flow. Shiqiao Station is the second station in the northbound direction of Line 3. A large number of residential buildings exist in the surrounding area. One-way trips have strong demand in the morning peak. The flow limiting station for the morning peak has a current limiting time of 7:30-9:30. According to the morning peak passenger flow distribution map of Shiqiao Station (Figure 8), the passenger flow around Shiqiao Station during the restricted period includes 23,000 people. The peak passenger flow period of this station is 7:30-8:30, and the total number of passengers is approximately 14,000 persons.

4.3. Calculation of Transfer Passenger Flow. According to the bus card data analysis, the passenger flow connection between Shiqiao Station and Zhujiang New Town Station is the closest in the morning peak hour, with a peak hour passenger flow of 1,467 persons. According to the passenger flow threshold of the diversion, the pair of rail stations satisfy the passenger flow conditions for diversion bus operation.

In terms of the travel time, the high-speed South China Expressway is selected for the diversion bus line, and the travel time is calculated based on the road speed during peak hours. The total travel time of the diversion line is approximately $60 \mathrm{~min}$. The total travel time of the original rail transit is approximately $65 \mathrm{~min}$, and the travel time difference is $-5 \mathrm{~min}$. The travel cost from Shiqiao Station to Zhujiang New Town Station is 6 Yuan. The average cost per trip is approximately 5.2 Yuan, considering a $40 \%$ discount after using the Yangchengtong bus card 15 times. In terms of travel security, the travel safety difference is zero, given that both travel modes have strict policies and effective measures for COVID-19 prevention and control and the safety of both travel modes are guaranteed. The diversion model adopts a commercial bus because the route is a long-distance diversion route connecting the central and suburban areas. 


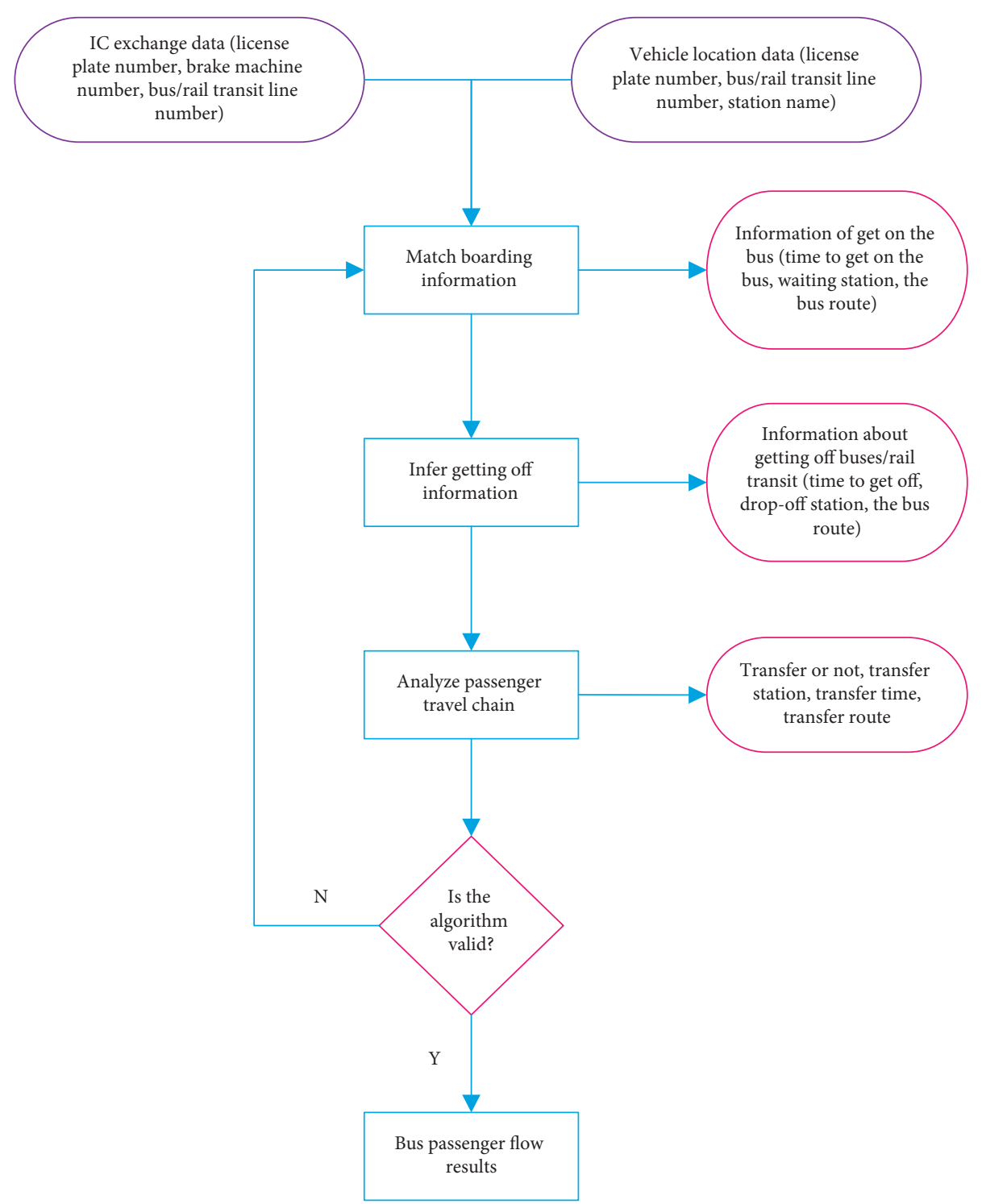

Figure 7: Main technical routes for bus travel analysis.

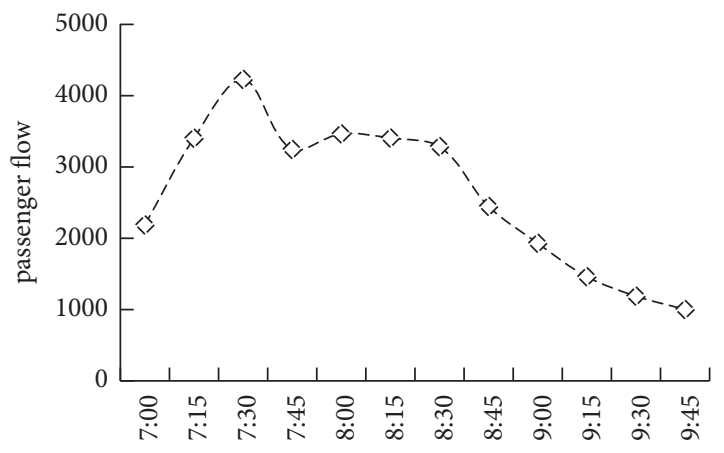

FIgURe 8: Time distribution of passenger flow at Shiqiao Station in the morning peak.

Based on the fares of the diversion routes that have been operated, the single fare is set as 7 Yuan, and the travel cost difference is -1.8 Yuan. In terms of the transfer characteristics, the survey data statistics show that the proportion of passengers who prefer bus transfer to those who prefer rail transit at Shiqiao Station is approximately 9.5\%. In addition, the number of transfer passengers during peak hours is 139 persons/h, and most passengers do not perform a transfer trip. Based on these conditions, the transfer passenger flow is 148 person-times/h in peak hours, accounting for $10.1 \%$ of the total passenger flow. This finding is in line with the actual survey transfer ratio. The transfer passenger flow calculation is shown in Table 8 .

4.4. Diversion Line Design. Diversion lines are designed on the basis of the previously described data analysis. The network signaling data during May 2020 are collected. The average daily number of users is 6.2 million, the average daily number of network signaling items is 2.1 billion, and the total number of monthly network signaling items is 64.3 billion. The accuracy and scale of the data satisfy the design requirements of the study. 
TABle 8: Transfer passenger flow calculation.

\begin{tabular}{lcc}
\hline Category & Transfer passengers & Direct passengers \\
\hline Passenger flow & 139 & 1,328 \\
Travel time difference & -5 & -5 \\
Travel cost difference & -1.8 & -1.8 \\
Travel security difference & 0 & 0 \\
Vehicle comfort & 1 & 1 \\
Transfer characteristics & 1 & 0 \\
Transition probability & $71.5 \%$ & $3.7 \%$ \\
Transfer passenger flow & 99.4 & 49.0 \\
\hline
\end{tabular}

Through network phone signaling data, the source of the inbound passenger flow of the rail station and distribution of the passenger flow after entering the station through a specific rail transit station are obtained to explore the OD distribution of the origin of passengers. From Shiqiao Station to Zhujiang New Town Station, the passenger flow near the original station is mainly distributed in the areas north of Shiqiao Station, such as Baiyue Square, Xincheng Xipian, Beicheng Baiyun Garden, and other communities. In addition, the passenger flow near the destination station is mainly centered in regions near Huacheng Square, such as Autoland Plaza. The specific passenger flow distribution and intensity are shown in Figure 9.

The starting and ending pickup points with a relatively large passenger flow are selected as the diverging OD points to connect the passenger flow, considering the parking conditions around the origin and destination stations. No intermediate station exists. The "one person, one seat" highquality bus is adopted to ensure that the passenger flow is point-to-point, fast, and direct because the expressway connects the OD points.

According to these principles, a "door-to-door" diversion route is planned to increase the attractiveness of the diversion route. The starting pickup point is set in a large community, and the ending pickup point is set in an office building that is a destination for many employees. The planned diversion route from Shiqiao to Zhujiang New Town is as follows: starting pickup point (South Gate of Baiyue Plaza, Xincheng West, Beicheng Baiyun Garden, and Laifu Garden) and ending pickup point (Gold Land Plaza), mainly via the Guangming North Road-Panyu AvenueSouth China Expressway-Huacheng Avenue-Pingjiang Road-Linjiang Avenue path. The transfer passenger flow along the line is 91 passengers/h. Two 50 -seat commercial bus diversion routes are organized at 7:30 and 8:00 at the peak passenger flow. The average full load rate is $91 \%$, which has relatively high economic benefits. The conceptual diagram of the specific route organization mode is shown in Figure 10.

\section{Discussion}

Urban rail transit has the advantages of large volume, fast speed, security and comfort, energy saving, and environmental protection. As a result, the transfer from low-quality and low-speed modes to high-quality and high-speed modes becomes a general and regular passenger flow transfer phenomenon. Therefore, considerable research has currently focused on the transfer of passenger flow from buses to newly built rail transit systems. However, the problem involving the diversion of the passenger flow from rail transit to buses is considerably different from the transfer problem.

Based on a stated preference survey, we comprehensively analyzed residents' travel psychology, the advantages and disadvantages of rail transit and conventional buses, travel time, travel cost, travel safety, and vehicle comfort. We also investigated the relationship between the relevant influencing factors and transition probability from rail transit to buses. Based on the data obtained from the survey, a binary Logit model for diversion transfer was constructed, and the decision tree was applied to the prediction of diversion transfer. In addition, based on the stochastic utility theory, a rail transit diversion probability model was constructed to provide a theoretical basis and technical support for the planning of rail transit diversion routes in related cities.

At present, the development stages of urban rail transit in various cities are relatively different, and evident differences are observed in the passenger flow. In some cities that have entered a mature operation stage, such as Beijing, Shanghai, and Guangzhou, the problem of congestion in the carriages during peak hours has become increasingly prominent, which has led to control of the flow of some stations. The passenger flow control has reduced the service level of rail transit. In this regard, many cities have attempted to realize diversion, but the implementation has not been effective due to the lack of theoretical support. Therefore, we comprehensively considered the relative factors that affect the travel mode preference of passengers and relative advantages of buses. We found that the travel time was the most significant factor affecting passenger flow transfer, followed by the vehicle comfort. Furthermore, Guangzhou Rail Transit Line 3 was considered an example, and the diversion route planning and design were performed according to the constructed diversion transfer probability model to verify the effectiveness and practicability of the model. The research provided an effective theoretical basis and technical reference for other cities to perform rail traffic diversion planning.

Research results show that travel time has the most significant impact on travel mode preference. Therefore, the organization of public transportation routes, delivery volume, and travel speed outside should be improved. Moreover, setting up bus lanes, optimizing bus routes, and rationally planning stations are also conducive to increasing the speed of urban buses and ensuring the transportation capacity and efficiency of buses. In addition, vehicle comfort and travel security also play a key role in people's travel mode preference. The transportation management should focus on the comprehensive function development and hardware support of public transportation stations. The convenience and comfort of rail transit are closely related to the facilities and functions of the stations and their connections, which should be highly valued. A reasonable and scientific bus internal layout is essential, such as improving the comfort of bus seats; rationally arranging seats to make full use of the internal space of the bus; improving the 


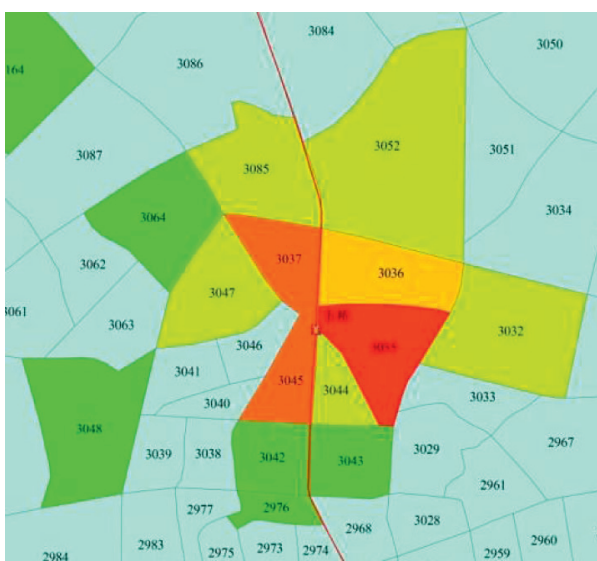

(a)

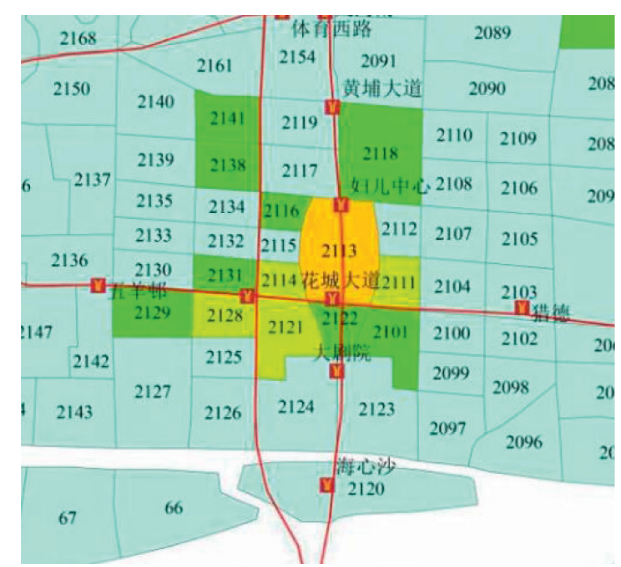

(b)

Figure 9: Origin and destination of passenger flow at Shiqiao Station. (a) Origin of the passenger flow. (b) Destination of the passenger flow.

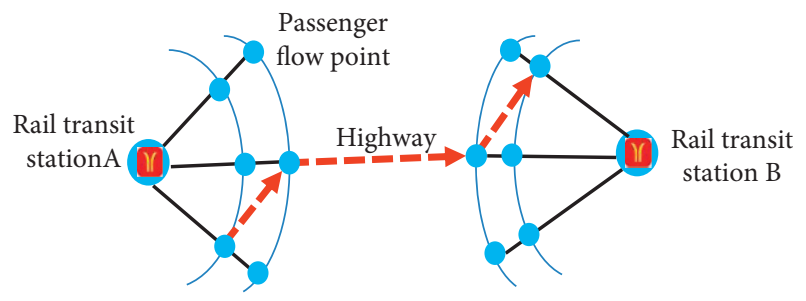

Figure 10: Conceptual diagram of line organization mode.

armrests of the rear seats and increasing the practical area of the armrests; improving the external details of the bus to increase the beauty of the body. At the same time, in order to improve travel security, it is necessary to deepen the reform of the bus driver training mechanism, strengthen the training of drivers' awareness of potential hazards, improve the incentive system, and standardize driving operations. Certain penalties shall be imposed on behaviors of occupying bus lanes for driving and illegal parking so as to improve the operating environment of buses, strengthen safety supervision on special road sections, gradually improve traffic signal facilities, separate motorized and nonmotorized vehicles, increase supervision, and improve lighting conditions. The combined operation and integrated development of bus and rail transportation are very important. The transfer connection between the bus station and the rail transportation station can be strengthened so that the two stations can complement each other during peak hours. Two relatively independent bus routes can also be established to strengthen the transfer connection between conventional bus stations and rail transportation stations.

\section{Conclusions}

The main conclusions drawn are as follows:

(1) Five influencing factors are selected to conduct a multiscenario SP travel willingness survey, and the relationship between the influencing factors and transition probability is analyzed. The results showed that the travel time influences the travel mode preference most significantly, followed by the vehicle comfort and security, whereas the impact of the travel costs and transfer characteristics is less significant.

(2) Based on the theory of the random utility, a conceptual model of diversion transfer Logit model is constructed, and the maximum likelihood estimation method is used to calibrate the parameters. Guangzhou Shiqiao Station is considered an example of diversion route planning and design, and the validity and practicability of the model are verified.

Based on the derived results, suggestions can be made as follows: (1) The organization of public transportation routes, delivery volume, and travel speed outside should be improved. For example, the operators of public transportation should maintain a close watch on the extreme weather and adjust the operation plan in time. (2) The operation of bus and rail transportation should be combined with integrated development. Some cities have established mechanisms that are worthy of promotion, including the transfer benefit between rail transit and buses. (3) The transportation management should focus on the comprehensive function development and hardware support of public transportation stations. The convenience and comfort of rail transit are closely related to the facilities and functions of the stations and their connections, which should be highly valued. For example, the seamless transfer between rail transit and buses may be preferred in most cities.

The conclusions involve the relationship between the five influencing factors and transition probability and the conceptual model of the diversion transfer Logit model based on the theory of the random utility. The conclusions can be applied to some cities, such as Shanghai, where rail transit has high passenger flow and great rail transit development. For example, Shanghai Rail Transit Line 2 has the highest passenger flow in Shanghai rail transit lines, and some stations, such as Lujiazui, Nanjing East Road, People's Square, Nanjing West Road, Jing'an Temple, Jiangsu Road, and Zhongshan Park, have relatively large passenger flow connection. However, for some cities with immature 
development stages of rail transit and some cities or lines with lower passenger flow, the conclusions may not be applied because the small number of survey samples may cause flawed representation of the sample data. The possible solution is to adopt a larger sample size to ensure that the sample data are more representative. In addition, the calculation granularity of the diversion passenger flow can be reduced according to actual requirements to render more targeted results.

The representation of the sample data may be flawed due to the small number of survey samples. Future work may aim to adopt a larger sample size to ensure that the sample data are more representative. In addition, the calculation granularity of the diversion passenger flow can be reduced according to actual requirements. For example, the granularity of $10 \mathrm{~min}$ or $15 \mathrm{~min}$ may render more targeted calculation results.

\section{Data Availability}

The data can only be shared internally within the institute where the first author works.

\section{Conflicts of Interest}

The authors declare that they have no conflicts of interest.

\section{Acknowledgments}

This work was supported by the National Natural Science Foundation of China (71803110), "Chen Guang" project of the Shanghai Municipal Education Commission, and Shanghai Education Development Foundation (19CG41).

\section{References}

[1] http://www.ecrrc.com/news/zxzt/3.html. 2021.8.10.

[2] W. Li, Q. Peng, C. Wen, S Li, X Yan, and X Xu, "Integrated optimization on energy saving and quality of service of urban rail transit system," Journal of Advanced Transportation, vol. 2020, Article ID 3474020, 22 pages, 2020.

[3] G. Yong and H. J. Huang, "Modal split in a competitive system of transit and highway withe heterogeneous users," Systems Engineering-Theory\&Practice, vol. 36, no. 9, pp. 2320-2327, 2016.

[4] G. Xiao, R. Wang, C. Zhang, and A. Ni, "Demand prediction for a public bike sharing program based on spatio-temporal graph convolutional networks," Multimedia Tools and Applications, vol. 80, no. 15, pp. 22907-22925, 2021.

[5] Z. H. Cheng, M. TRéPANIER, and L. J. Sun, "Incorporating travel behavior regularity into passenger flow forecasting," Transportation Research Part C, vol. 128, p. 128, 2021.

[6] H. Z. Zhu, X. G. Yang, and Y. Z. Wang, "Prediction of daily entrance and exit passenger flow of rail transit stations by deep learning method," Journal of Advanced Transportation, vol. 2018, Article ID 6142724, 11 pages, 2018.

[7] X. Chen, H. Chen, Y. Yang et al., "Traffic flow prediction by an ensemble framework with data denoising and deep learning model," Physica A: Statistical Mechanics and Its Applications, vol. 565, Article ID 125574, 2021.

[8] X. D. Ma, Y. K. Yang, and G. D. Xia, "Research on the relationship between the characteristics of urban rail transit passenger flow and the development stage," in Proceedings of the Proceedings of the 2020 China Urban Transport Planning Annual Conference, Beijing, China, December 2020.

[9] Q. Shen, P. Chen, and H. Pan, "Factors affecting car ownership and mode choice in rail transit-supported suburbs of a large Chinese city," Transportation Research Part A: Policy and Practice, vol. 94, pp. 31-44, 2016.

[10] M. W. Xin, A. Shalaby, S. M. Feng, and H Zhao, "Impacts of COVID-19 on urban rail transit ridership using the Synthetic Control Method," Transport Policy, vol. 111, p. 111, 2021.

[11] R. Lu, "Does investing in rail transit benefit the poor? A comparative study of rail and bus travel by low-income households in the California household travel survey," Transportation Research Record: Journal of the Transportation Research Board, vol. 2672, no. 6, pp. 11-20, 2018.

[12] X. Wang, Y. Wang, Z. Zhang, and J Li, "Do rail transits improve local air quality? Take Chengdu-Nanchang for example," Air Quality, Atmosphere \& Health, vol. 14, 2021.

[13] J. H. Hu, Z. Huang, Z. F. Cheng, and D Jun, "Analysis on space-time benefit change of bus passengers' shopping in commercial center," Journal of Chongqing Jianzhu University, vol. 34, no. 06, pp. 101-105+110, 2015.

[14] X. Y. Cheng, Y. Cao, K. Huang, and Y Wang, "Modeling the satisfaction of bus traffic transfer service quality at a highspeed railway station," Journal of Advanced Transportation, vol. 2018, Article ID 7051789, 12 pages, 2018.

[15] J. Du, F. Li, J. Li et al., "Evaluating the technological evolution of battery electric buses: China as a case," Energy, vol. 176, pp. 309-319, 2019.

[16] M. Mahmoud, R. Garnett, M. Ferguson, and P. Kanaroglou, "Electric buses: a review of alternative powertrains," Renewable and Sustainable Energy Reviews, vol. 62, pp. 673-684, 2016.

[17] J. Cui, Y. E. Gao, J. Cheng, and L Shi, "Study on the selection model of staying adjustment bus lines along rail transit," Journal of Advanced Transportation, vol. 2020, Article ID 6385359, 12 pages, 2020.

[18] A. Lozano, "Special issue on "Urban transportation sustainability: experiences and innovative approaches"," Transportation Research Part A, vol. 137, 2020.

[19] X. N. Dong, Z. C. Yang, H. M. Zhao, and H Ji, “Analysis on travel mode choice behavior based on spatial-temporal constraint," Journal of Highway and Transportation Research and Development, vol. 37, no. 09, pp. 104-112, 2020.

[20] L. Böcker, M. Dijst, and J. Faber, "Weather, transport mode choices and emotional travel experiences," Transportation Research Part A: Policy and Practice, vol. 94, no. dec, pp. 360-373, 2016.

[21] L. J. Tian, Q. Yang, H. J. Huang, and C. R. Lv, “The cumulative prospect theory-based travel model choice model and its empirical verification," Systems EngineeringTheory \& Practice, vol. 36, no. 7, 2016.

[22] S. Agarwal, M. Diao, J. Keppo, and T. F. Sing, "Preferences of public transit commuters: evidence from smart card data in Singapore," Journal of Urban Economics, vol. 120, Article ID 103288, 2020.

[23] J. De Vos, P. L. Mokhtarian, T. Schwanen, V. Van Acker, and F. Witlox, "Travel mode choice and travel satisfaction: bridging the gap between decision utility and experienced utility," Transportation, vol. 43, no. 5, pp. 771-796, 2016.

[24] T. Nordfjærn, Ö ŞIMŞEKOĞLU, Ö Simşekoğlu, H. B Lind, S. H Jørgensen, and T Rundmo, "Transport priorities, risk perception and worry associated with mode use and preferences among Norwegian commuters," Accident Analysis \& Prevention, vol. 72, pp. 391-400, 2014. 
[25] P. A. Singleton and L. Wang, "Safety and security in discretionary travel decision making: focus on active travel mode and destination choice," Transportation Research Record Journal of the Transportation Research Board, vol. 2430, no. 2430, pp. 47-58, 2014.

[26] Y. Wen, J. Leng, X. Shen, G Han, L Sun, and F Yu, "Environmental and health effects of ventilation in subway stations: a literature review," International Journal of Environmental Research and Public Health, vol. 17, no. 3, 2020.

[27] B. Aaditya and T. M. Rahul, "Psychological impacts of COVID-19 pandemic on the mode choice behaviour: a hybrid choice modelling approach," Transport Policy, vol. 108, pp. 47-58, 2021.

[28] M. Khadem Sameni, A. Barzegar Tilenoie, and N. Dini, "Will modal shift occur from subway to other modes of transportation in the post-corona world in developing countries?" Transport Policy, vol. 111, pp. 82-89, 2021.

[29] P. Loa, S. Hossain, S. M. Mashrur et al., "Exploring the impacts of the COVID-19 pandemic on modality profiles for non-mandatory trips in the Greater Toronto Area," Transport Policy, vol. 110, pp. 71-85, 2021.

[30] C. Eisenmann, C. Nobis, V. Kolarova, B. Lenz, and C. Winkler, "Transport mode use during the COVID-19 lockdown period in Germany: the car became more important, public transport lost ground," Transport Policy, vol. 103, pp. 60-67, 2021.

[31] S. G. Hu, R. H. Wang, X. X. Wang, and Y. Y. Liu, "Modeling of travel mode choice behavior of residents in different stages of the COVID-19 epidemic," Journal of Guangdong University of Technology, vol. 38, no. 01, pp. 32-38, 2021.

[32] N. Jiang, S. Li, S. Z. Cao et al., "Transportation activity pattens of Chinese population during COVID-19 epidemic," Research of Environmental Sciences, vol. 33, no. 07, p. 8, 2020.

[33] X. P. Yang, "Shanghai traffic operation characteristics under the influence of COVID-19," Traffic \& Transportation, vol. 37, no. 3, p. 5, 2021.

[34] T. Lv, Study on Passenger Flow Diversion between Regular Public Transit and Proposed Rail Transit Based on Discrete Choice Model, Chang'an University, Xi'an, 2011.

[35] P. H. Zhang, Study of Travel Model Shift between Regular Public Bus and Urban Rail Transit, Chang'an University, Xi'an, 2012.

[36] J. Q. Cao, L. X. Wang, X. H. Sun, and L. N. Zhao, "Study of passenger flow shift of urban rail transit based on Nested Logit model," China Sciencepaper, vol. 12, no. 7, pp. 749-753, 2017.

[37] M. He, X. Guo, J. Ran, C. Wu, W. Zhu, and C. Liu, "Forecasting rail transit split with disaggregated MNL model," Journal of Transportation Systems Engineering and Information Technology, vol. 10, no. 2, pp. 136-142, 2010.

[38] S. Flügel, A. H. Halse, J. d. D. Ortúzar, and L. I. Rizzi, "Methodological challenges in modelling the choice of mode for a new travel alternative using binary stated choice data - the case of high speed rail in Norway," Transportation Research Part A: Policy and Practice, vol. 78, no. AUG, pp. 438-451, 2015.

[39] P. Marcel, T. Dirk, V. Akshay, and W JoanL, "Values, attitudes and travel behavior: a hierarchical latent variable mixed logit model of travel mode choice," Transportation, vol. 41, no. 4, pp. 873-888, 2014.

[40] R. R. Yang and R. Y. Long, "Affecting factor of theoretical model in urban residents green travel behavior based on grounded theory," Wuhan University Journal (philosophy \& Social Sciences), vol. 67, no. 05, pp. 13-19, 2014.

[41] S. P. Wu, L. J. Tian, and Y. F. Fang, "Study on the travel mode choice of commuters in an activity based model," Journal of Systems Science and Mathematical Sciences, vol. 40, no. 10, 2020. 